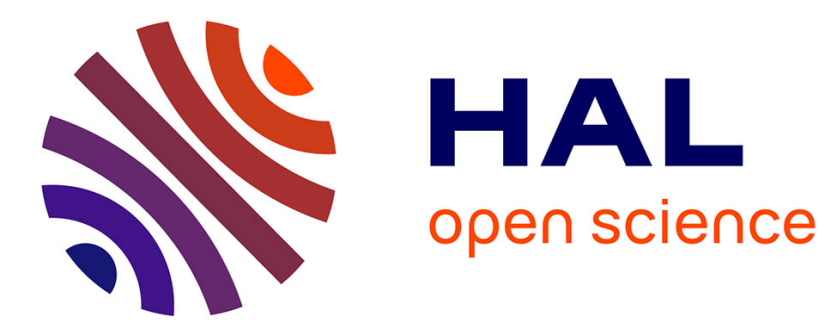

\title{
Configuration-seniority spectral distributions in Ni62
}

S. Spitz, C. Quesne

\section{To cite this version:}

S. Spitz, C. Quesne. Configuration-seniority spectral distributions in Ni62. Journal de Physique Lettres, 1976, 37 (1), pp.3-6. 10.1051/jphyslet:019760037010300 . jpa-00231224

\section{HAL Id: jpa-00231224 https://hal.science/jpa-00231224}

Submitted on 1 Jan 1976

HAL is a multi-disciplinary open access archive for the deposit and dissemination of scientific research documents, whether they are published or not. The documents may come from teaching and research institutions in France or abroad, or from public or private research centers.
L'archive ouverte pluridisciplinaire HAL, est destinée au dépôt et à la diffusion de documents scientifiques de niveau recherche, publiés ou non, émanant des établissements d'enseignement et de recherche français ou étrangers, des laboratoires publics ou privés. 


\title{
CONFIGURATION-SENIORITY SPECTRAL DISTRIBUTIONS IN $\mathbf{N i}^{62}$
}

\author{
S. SPITZ $\left({ }^{*}\right)$ and C. QUESNE $(* *)$ \\ Physique Théorique et Mathématique, Université Libre de Bruxelles, Bruxelles, Belgique
}

(Reçu le 8 septembre 1975, accepté le 15 octobre 1975)

\begin{abstract}
Résumé. - Les distributions spectrales des configurations à séniorité fixée sont utilisées pour analyser quelques propriétés de $\mathrm{Ni}^{62}$ et les résultats obtenus sont comparés à ceux du modèle en couches. La validité de la symétrie symplectique est discutée à partir des distributions des états à séniorité fixée dans chaque orbite.
\end{abstract}

Abstract. - Configuration-seniority spectral distributions are used to analyse some properties of $\mathrm{Ni}^{62}$, and the results compared with those of shell model. The goodness of the symplectic symmetry is discussed by investigating the distributions with fixed seniority in every orbit.

During the last few years significant progress has been made in the study of the spectra of complex nuclei involving a large number of configurations, by the introduction of the spectral distribution method [1-3]. Applications of this method to the calculation of low excitation spectra and level densities and to the study of symmetries have been carried out in some cases.

In a series of papers we intend to study the symplectic symmetry, connected with the seniority quantum number, for systems of $n$ identical nucleons distributed in a finite set of levels with total degeneracy $2 \Omega$. In a former work [4] we studied fixed generalized seniority distributions, associated with the chain of groups $V(2 \Omega) \supset \mathrm{Sp}(2 \Omega)$. In this paper we give preliminary results for configuration-seniority distributions, connected with the chain of groups

$$
V(2 \Omega) \supset \sum_{a} \oplus V\left(2 \Omega_{a}\right) \supset \sum_{a} \oplus \operatorname{Sp}\left(2 \Omega_{a}\right),
$$

wherein the averages are taken over subspaces characterized by

$$
[\mathrm{nv}]=\left[n_{a} v_{a} n_{b} v_{b} \ldots\right], \quad \sum_{a} n_{a}=n
$$

$n_{a}$ and $v_{a}$ being the number of particles and the seniority in orbit $a$ respectively. The states belonging to these $d([\mathbf{n v}])$ dimensional subspaces are denoted by $|[\mathbf{n v}] \xi\rangle$, where $\xi$ stands for all the quantum numbers necessary to complete the classification. Here we

$\left({ }^{*}\right)$ Chercheur agréé I.I.S.N.

(**) Maître de recherches F.N.R.S. restrain ourselves to discuss the $\mathrm{Ni}$ isotopes where a shell model exact calculation can be made $\left({ }^{1}\right)$, as a necessary step on the way of using the method for more complex nuclei. In particular we analyse $\mathrm{Ni}^{62}$ in a three level model $\left[2 p_{3 / 2}, 1 f_{5 / 2}, 2 p_{1 / 2}\right]^{6}$, using the Auerbach residual interaction [5].

The centroid energies :

$$
\mathcal{E}([\mathbf{n v}])=d^{-1}([\mathbf{n v}]) \sum_{\xi}\langle[\mathbf{n v}] \xi|H|[\mathbf{n v}] \xi\rangle,
$$

as well as the total widths

$$
\begin{aligned}
\mu_{2}([\mathbf{n v}]) & =\sigma^{2}([\mathbf{n v}])= \\
= & d^{-1}([\mathbf{n v}]) \sum_{\xi}\left\langle[\mathbf{n v}] \xi\left|[H-\varepsilon([\mathbf{n v}])]^{2}\right|[\mathbf{n v}] \xi\right\rangle \\
= & \sum_{\left[\mathbf{n}^{\prime} \mathbf{v}^{\prime}\right]} \sigma^{2}\left([\mathbf{n v}] \rightarrow\left[\mathbf{n}^{\prime} \mathbf{v}^{\prime}\right]\right)
\end{aligned}
$$

and the partial ones

$$
\begin{aligned}
\sigma^{2}\left([\mathbf{n v}] \rightarrow\left[\mathbf{n}^{\prime} \mathbf{v}^{\prime}\right]\right)=d^{-1}([\mathbf{n v}]) & \sum_{\xi \xi^{\prime}} \times \\
& \times\left|\left\langle\left[\mathbf{n}^{\prime} \mathbf{v}^{\prime}\right] \xi^{\prime}|H|[\mathbf{n v}] \xi\right\rangle\right|^{2} \\
& -\delta_{[\mathbf{n v}],\left[\mathbf{n}^{\prime} \mathbf{v}^{\prime}\right]}[\mathcal{E}([\mathbf{n v}])]^{2}
\end{aligned}
$$

are obtained by generalizing to $k$ levels the propagation laws of operator averages defined in a single orbit. General expressions will be published elsewhere, together with results for $\mathrm{Sn}$ and $\mathrm{Pb}$ nuclei.

Using the first two moments of the configuration-

( $\left.{ }^{1}\right)$ The shell model exact calculations reported in this paper were performed in Orsay with the Oak-Ridge-Rochester shell model code. 
seniority distributions, we build a two-moment (2M) approximation [2] :

$$
\begin{aligned}
\bar{F}(n, E) & =\sum_{[\mathbf{n v}]} \bar{F}([\mathbf{n v}], E) \\
& =\sum_{[\mathbf{n v}]} d([\mathbf{n v}])(2 \pi)^{-1 / 2} \int_{-\infty}^{x} \mathrm{e}^{-1 / 2 x^{\prime 2}} \mathrm{~d} x^{\prime}, \\
x & =[E-\delta([\mathbf{n v}])] / \sigma([\mathbf{n v}]),
\end{aligned}
$$

of the exact distribution function

$$
F(n, E)=\sum_{\substack{i=0 \\ E_{i} \leqslant E}}^{j}\left(2 J_{i}+1\right) .
$$

We call it the $2 \mathrm{M}$ configuration-seniority distribution function.

As the dimensionalities that we have to deal with in $\mathrm{Ni}^{62}$ are small $(1 \leqslant d([\mathrm{nv}]) \leqslant 140)$, the $2 \mathrm{M}$ approximation might be a poor one. This drawback always arises when a fine subdivision of the total space is made. However it is particularly dramatic for the seniority representation, as there are always subspaces of dimension 1 , whatever large the total space may be. For this reason we also use the four-moment (4M) configuration-seniority distribution function [2] :

$$
\begin{aligned}
\bar{F}(n, E)= & \sum_{[\mathbf{n v}]} \bar{F}([\mathbf{n v}], E)= \\
& =\sum_{[\mathbf{n v}]} d([\mathbf{n v}])(2 \pi)^{-1 / 2} \int_{-\infty}^{x} \times \\
& \times\left\{\mathrm{e}^{-1 / 2 x^{\prime 2}} \times\left[1+\frac{1}{6} \gamma_{1}([\mathbf{n v}])\left(x^{\prime 3}-3 x^{\prime}\right)\right.\right. \\
& \left.\left.+\frac{1}{24} \gamma_{2}([\mathbf{n v}])\left(x^{\prime 4}-6 x^{\prime 2}+3\right)\right]\right\} \mathrm{d} x^{\prime}, \quad
\end{aligned}
$$

with values of the skewness $\gamma_{1}=\mu_{3} \mu_{2}^{-3 / 2}$, and the excess $\gamma_{2}=\mu_{4} \mu_{2}^{-2}-3$ computed from the $n$ particle $H$ matrix elements. These values are large

$$
\left(-1.1 \leqslant \gamma_{1} \leqslant 1.1 ;-1.1 \leqslant \gamma_{2} \leqslant 1.8\right),
$$

showing distributions rather far from the normal form.

The exact distribution function is well reproduced by the $4 \mathrm{M}$ approximation defined in eq. (6). However it turns out that the $4 \mathrm{M}$ configuration distribution function [1], i.e. the analog of eq. (6) for configuration distributions, [nv] being replaced everywhere by $[\mathrm{n}]=\left[n_{a} n_{b} \ldots\right]$, can do almost as well, and moreover is better than the $2 \mathrm{M}$ configuration-seniority distribution function given by eq. (4). Therefore, in our case, the distribution is more improved by using higher moments than by taking a finer subdivision of the total space.

The improvement in going from $2 \mathrm{M}$ to $4 \mathrm{M}$ distribution is especially clear at low energy, as is shown on the spectra of figure 1, calculated using Ratcliff's prescription [2]. The r.m.s. deviation between the exact absolute energies of the first 12 excited states and those obtained with the $2 \mathrm{M}$ and $4 \mathrm{M}$ [nv] approxi- mations is 0.48 and $0.16 \mathrm{MeV}$ respectively. In this comparison we exclude the ground state which is drastically shifted up in the $4 \mathrm{M}$ approximation. Binding energies referred to the second excited state, i.e. $E_{2}-\left(E_{2}^{\exp }-E_{0}^{\exp }\right)$, are $-0.95,-0.35$ and $-0.29 \mathrm{MeV}$ for the $2 \mathrm{M}$ and $4 \mathrm{M}$ [nv] approximations, and the exact calculation respectively.

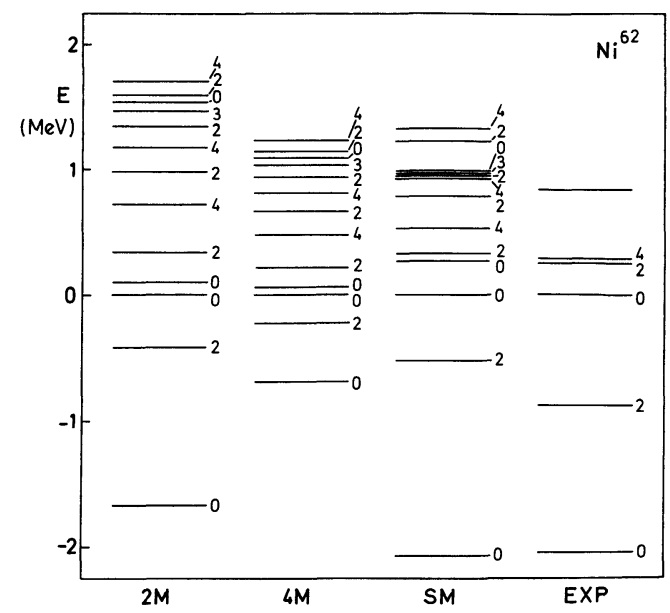

Fig. 1. - Comparison between the exact shell model and 2M and $4 \mathrm{M}$ configuration-seniority spectra of $\mathrm{Ni}^{62}$. The experimental spectrum is also shown. The second excited level is taken as a reference point.

A finer comparison with the exact shell model calculation can be made by considering the structure of the low lying eigenvectors. For this purpose, we evaluate the summed intensity :

$$
\sum_{i=0}^{5} \sum_{\xi}\left|\left\langle[\mathbf{n v}] \xi \mid \psi^{(i)}\right\rangle\right|^{2},
$$

of the various [nv] subspaces in the 6 lowest eigenstates $\left|\psi^{(i)}\right\rangle, i=0,1, \ldots, 5$, using the partial distribution functions $\bar{F}([\mathbf{n v}], E)$ and Ratcliff's prescription [2]. The r.m.s. deviation between the exact and the $2 \mathrm{M}$ (or $4 \mathrm{M}$ ) results is found to be 0.79 (or 0.49 ). The

\begin{tabular}{|c|c|c|c|c|c|}
\hline \multicolumn{2}{|c|}{$2 \mathrm{M}$} & \multicolumn{2}{|c|}{$4 \mathrm{M}$} & \multicolumn{2}{|c|}{ S.M. } \\
\hline [nv] & Intensity & {$[\mathbf{n v}]$} & Intensity & {$[\mathbf{n v}]$} & Intensity \\
\hline 1 22 11 ] & 12.36 & {$\left[\begin{array}{lll}31 & 31 & 00\end{array}\right]$} & 14.72 & {$\left[\begin{array}{lll}31 & 31 & 00\end{array}\right]$} & 22.28 \\
\hline $\left.\begin{array}{lll}31 & 31 & 00\end{array}\right]$ & 11.71 & {$\left[\begin{array}{lll}31 & 22 & 11\end{array}\right]$} & 11.48 & {$\left[\begin{array}{lll}31 & 20 & 11\end{array}\right]$} & 8.98 \\
\hline$\left[\begin{array}{lll}22 & 33 & 11\end{array}\right]$ & 7.57 & {$\left[\begin{array}{lll}31 & 20 & 11\end{array}\right]$} & 8.38 & {$\left[\begin{array}{lll}31 & 22 & 11\end{array}\right]$} & 6.96 \\
\hline$\left[\begin{array}{lll}22 & 31 & 11\end{array}\right]$ & 7.56 & {$\left[\begin{array}{lll}22 & 31 & 11\end{array}\right]$} & 6.39 & {$\left[\begin{array}{lll}31 & 11 & 20\end{array}\right]$} & 6.20 \\
\hline$\left[\begin{array}{lll}31 & 33 & 00\end{array}\right]$ & 6.03 & {$\left[\begin{array}{lll}40 & 22 & 00\end{array}\right]$} & 5.59 & {$\left[\begin{array}{llll}1 & 3 & 31 & 20\end{array}\right]$} & 5.29 \\
\hline
\end{tabular}
decrease of this deviation in going from $2 \mathrm{M}$ to $4 \mathrm{M}$ is not due to an improvement of the whole structure as it is for the spectrum, but to that of a few particular results. For instance the intensity of the $[22,33,11]$ subspace in sharply decreased. As shown in table I,

\section{TABLE I}

Percentage intensities of the most important configuration-seniority subspaces in the 6 lowest lying eigenstates of $\mathrm{Ni}^{62}$. 
the ordering of the various subspaces in terms of their importance in the low lying eigenstates is not reproduced very well. It is significant that we have to use the 4M approximation in order to get the three most important intensities. The reason is that the exact relative intensities of [nv] subspaces vary too much from eigenvalue to eigenvalue for their averages over a few eigenstates to be reproduced with high accuracy by a continuous approximation.

When compared with Ratcliff's results [2], this behaviour is somewhat disappointing, although not completely unexpected. Indeed as we are not working with fixed $J$ distributions, all the subspaces do not contribute to each one of the 6 eigenstates, and this greatly perturbs the statistical description of the summed intensity. On the other hand, our results are somewhat worse than those obtained for other badly broken symmetries, such as configurations. This is a consequence of the selection properties of seniority with respect to angular momentum (for instance $v=0$ corresponds to $J=0$ only). Owing to these, there are nearby eigenstates with different angular momenta, which belong almost entirely to orthogonal sets of [nv] subspaces. This fact again does not favour a statistical description.

In order to study the goodness of the symplectic symmetry, we sum over $n_{a}, n_{b}, \ldots$ and get the centroids, and total and partial widths of fixed seniority distributions. The partial widths

$$
\begin{aligned}
\sigma^{2}\left([\mathbf{v}] \rightarrow\left[\mathbf{v}^{\prime}\right]\right)=d^{-1}([\mathbf{v}]) & \sum_{[\mathbf{n}],\left[\mathbf{n}^{\prime}\right]} \times \\
& \times d([\mathbf{n v}]) \sigma^{2}\left([\mathbf{n v}] \rightarrow\left[\mathbf{n}^{\prime} \mathbf{v}^{\prime}\right]\right), \\
d([\mathbf{v}]) & =\sum_{[\mathbf{n}]} d([\mathbf{n v}]),
\end{aligned}
$$

are listed in table II ; for most of the 12 [v] subspaces the internal widths $\sigma^{2}([\mathbf{v}] \rightarrow[\mathbf{v}])$ are larger than the external ones, $\sum_{\left[\mathbf{v}^{\prime}\right] \neq[\mathbf{v}]} \sigma^{2}\left([\mathbf{v}] \rightarrow\left[\mathbf{v}^{\prime}\right]\right)$.
It is in general admitted [6] that the perturbation parameter

$$
I^{2}\left([\mathbf{v}] \rightarrow\left[\mathbf{v}^{\prime}\right]\right)=\sigma^{2}\left([\mathbf{v}] \rightarrow\left[\mathbf{v}^{\prime}\right]\right) /\left[\mathcal{E}([\mathbf{v}])-\mathcal{E}\left(\left[\mathbf{v}^{\prime}\right]\right)\right]^{2}
$$

provides, when small and the centroids well separated, a good basis for space truncation. An analysis of the $I^{2}\left([\mathbf{v}] \rightarrow\left[\mathbf{v}^{\prime}\right]\right)$ table shows only one subspace splitting out, namely [000] $\left(I_{\max }^{2}=0.035\right)$; a diagonalization is made in this 6 dimensional subspace, and the approximate eigenstates so obtained, $\left|\psi_{\text {trunc }}\right\rangle$, are compared with the exact ones, $\left|\psi_{\text {exact }}\right\rangle$, which have the largest components in the [000] subspace.

Though the energies are found to lie close to the exact ones $\left(E_{\text {exact }}-E_{\text {trunc }}=-0.04,-0.06,-0.22\right.$, $-0.35,-0.08$ and $0.25 \mathrm{MeV}$ for the 6 eigenstates

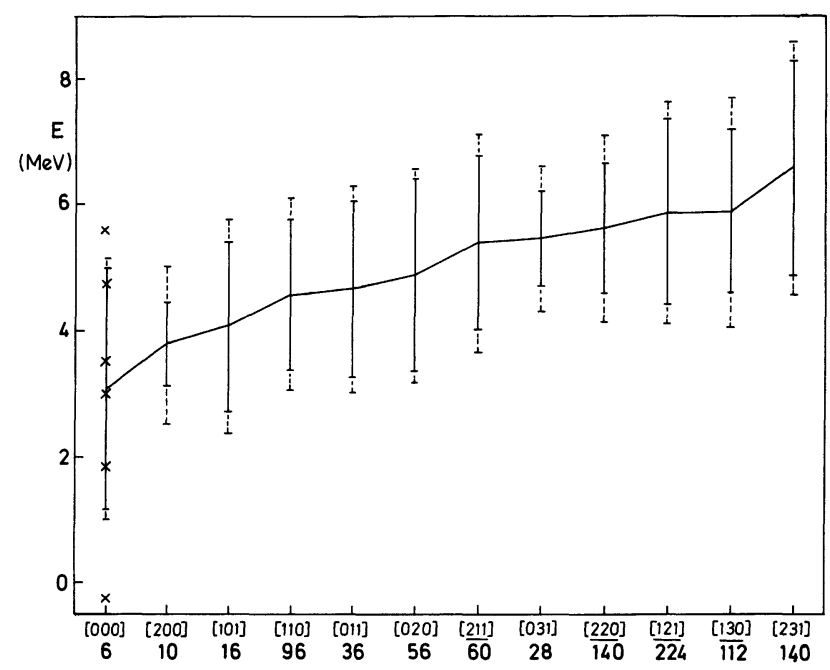

FIG. 2. - Centroid energies of the fixed seniority subspaces. The internal (solid line) and external widths (dashed line) are also plotted. The subspaces which mix with the [000] subspace are underlined. Under each subspace is written its dimension. The position of the eigenvalues resulting from a diagonalization in

\begin{tabular}{|c|c|c|c|c|c|c|c|c|c|c|c|c|}
\hline & [000] & [200] & [101] & [110] & [011] & [020] & [211] & [031] & {$\left[220^{\circ}\right]$} & [121] & [130] & [231] \\
\hline & & & & & 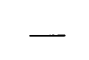 & - & - & - & - & - & - & \\
\hline & 3.647 & 0 & 0 & 0 & 0 & 0 & 0.090 & 0 & 0.224 & 0.263 & 0.003 & \\
\hline & 0 & 0.442 & 0.689 & 0.014 & 0.037 & 0.109 & 0.00 & & 0 & 0.209 & .003 & \\
\hline & 0 & 431 & 1.845 & .064 & .04 & .068 & 0.1 & 0.001 & 0.131 & & 0 & 0.0 \\
\hline & 0 & 002 & & 6 & & & 0.4 & & & & 0.063 & \\
\hline & 0 & & & & & & & 0 & 0.087 & & 0.0 & .16 \\
\hline & 0 & & & & & & 0.0 & & 0.096 & 0.150 & 0.094 & 0.102 \\
\hline & 0.009 & 001 & 0.0 & 0.730 & 0.009 & 0.032 & 1.90 & 0.075 & 0.043 & 0.178 & 0.127 & \\
\hline & 0 & 0 & & 30 & 0 & 0.0 & 0.1 & & 0.071 & 0.2 & 0.045 & \\
\hline & 010 & 0 & & & & & & & $1 .($ & & 0.070 & \\
\hline & 007 & & 0.010 & & & & & & & & & \\
\hline & 000 & 000 & & & & & 0.068 & 0.011 & 0.087 & 0.1 & 1.722 & \\
\hline & & 0 & 0.000 & 0.080 & 0.043 & 0.041 & 0 & 0 & 0.090 & 0.276 & 0.734 & \\
\hline
\end{tabular}
the [000] subspace is indicated by crosses.

TABLE II

Partial widths $\sigma^{2}\left([\mathbf{v}] \rightarrow\left[\mathbf{v}^{\prime}\right]\right)$. The ordering of $[\mathbf{v}]$ subspaces is according to increasing centroid energy 
in increasing energy order), some of the overlaps are very poor. We obtain indeed

$$
\left|\left\langle\psi_{\text {exact }} \mid \psi_{\text {trunc }}\right\rangle\right|^{2}=0.99,0.97,0.52,0.40,0.48
$$

and 0.52 respectively, and the results are similar when the Argonne interaction [7] is used instead of the Auerbach one.

The explanation of this situation can be seen on figure 2. The centroid energy of [000] is well separated from those of the other 4 subspaces which can mix with it. But the third eigenvalue is located at a distance less than $2 \sigma_{\text {int }}([211])$ from the centroid of the [211] subspace and in this range of energy the $J=0$ states belonging to [211] do not allow a good overlap between the exact and [000] eigenstates. As the truncation parameter $I^{2}\left([\mathbf{v}] \rightarrow\left[\mathbf{v}^{\prime}\right]\right)$ takes no account of the importance of the internal widths, its interpretation should be examined very carefully in each case. For the [000] subspace, it is clear that it can be used as a valuable criterium only in the very low part of the spectrum.

From the study of $\mathrm{Ni}^{62}$ that we have carried out in this work, we see that the configuration-seniority and seniority distributions are not so useful from the point of view of low-energy spectra and level densities as is might appear at first sight. Although the results could be better in heavier nuclei, we think that the main interest in computing such distributions in those nuclei lies in the determination of partial widths, and especially in the prediction of the admixing of other subspaces into the total seniority zero subspace.

One of us (S.S.) is indebted to Drs. Duchemin and Lorazo for discussions and help in solving some computational problems, and to the Division de Physique Théorique of the Institut de Physique Nucléaire, Orsay, for making available to him its computational facilities.

\section{References}

[1] French, J. B. and Ratcliff, K. F., Phys. Rev. C 3 (1971) 94.

[2] Ratcliff, K. F., Phys. Rev. C 3 (1971) 117.

[3] Chang, F. S., French, J. B. and Thio, T. H., Ann. Phys. 66 (1971) 137.

[4] Quesne, C. and SpITz, S., Ann. Phys. 85 (1974) 115.
[5] Auerbach, N., Phys. Rev. 163 (1967) 1203.

[6] Parikh, J. C. and Wong, S. S. M., Nucl. Phys. A 182 (1972) 593.

[7] Cohen, S., Lawson, R. D., Macfarlane, M. H., Pandya, S. P. and SogA, M., Phys. Rev. 160 (1967) 903. 